\title{
Erratum to: Statistical Study of ICMEs and Their Sheaths During Solar Cycle 23 (1996-2008)
}

\author{
E. Mitsakou • X. Moussas
}

Published online: 24 July 2014

(C) Springer Science+Business Media Dordrecht 2014

Erratum to: Solar Phys (2014) 289:3137-3157

DOI 10.1007/s11207-014-0505-y

The Appendix (Table 6) and the downloadable version available as Electronic Supplementary Material should be replaced with those given here. We regret the inconvenience.

The online version of the original article can be found under doi:10.1007/s11207-014-0505-y.

Electronic supplementary material The online version of this Erratum (doi:10.1007/s11207-014-0575-x) contains supplementary material, which is available to authorized users.

E. Mitsakou $(\varangle) \cdot X$. Moussas

Section of Astrophysics, Astronomy and Mechanics, Faculty of Physics, National and Kapodistrian University of Athens, Panepistimioupoli Zografou, 15783, Athens, Greece

e-mail: emitsaku@phys.uoa.gr

$\mathrm{X}$. Moussas

e-mail: xmoussas@phys.uoa.gr 


\section{Appendix}

Table 6 Catalog of ICMEs in 1996-2008.

\begin{tabular}{|c|c|c|c|c|c|c|c|c|c|}
\hline \multirow{2}{*}{$\begin{array}{l}\text { Driven } \\
\text { shock } \\
\mathrm{y}(\text { yes }) \text { or } \\
\mathrm{n}(\text { no) }\end{array}$} & \multicolumn{3}{|l|}{ Start of the sheath } & \multicolumn{3}{|l|}{ Start of the ICME } & \multicolumn{3}{|l|}{ End of the ICME } \\
\hline & day & hour & $\min$ & day & hour & $\min$ & day & hour & $\min$ \\
\hline $\mathrm{n}$ & 27 May 1996 & 12 & 37 & 27 May 1996 & 15 & 6 & 28 May 1996 & 23 & 45 \\
\hline $\mathrm{y}$ & 01 July 1996 & 14 & 1 & 01 July 1996 & 19 & 1 & 02 July 1996 & 9 & 41 \\
\hline $\mathrm{n}$ & 07 August 1996 & 8 & 44 & 07 August 1996 & 13 & 11 & 08 August 1996 & 5 & 31 \\
\hline $\mathrm{n}$ & 24 December 1996 & 1 & 19 & 24 December 1996 & 3 & 8 & 25 December 1996 & 11 & 50 \\
\hline $\mathrm{y}$ & 10 January 1997 & 0 & 56 & 10 January 1997 & 4 & 50 & 11 January 1997 & 3 & 12 \\
\hline $\mathrm{y}$ & 09 February 1997 & 13 & 18 & 10 February 1997 & 3 & 11 & 10 February 1997 & 19 & 8 \\
\hline $\mathrm{y}$ & 10 April 1997 & 17 & 40 & 11 April 1997 & 6 & 39 & 11 April 1997 & 20 & 33 \\
\hline $\mathrm{n}$ & 21 April 1997 & 11 & 9 & 21 April 1997 & 12 & 57 & 23 April 1997 & 0 & 28 \\
\hline $\mathrm{y}$ & 15 May 1997 & 2 & 3 & 15 May 1997 & 10 & 38 & 16 May 1997 & 0 & 12 \\
\hline y & 20 May 1997 & 6 & 2 & 20 May 1997 & 15 & 34 & 21 May 1997 & 4 & 37 \\
\hline $\mathrm{y}$ & 23 May 1997 & 23 & 52 & 24 May 1997 & 15 & 23 & 25 May 1997 & 7 & 52 \\
\hline $\mathrm{y}$ & 25 May 1997 & 14 & 33 & 25 May 1997 & 19 & 59 & 26 May 1997 & 9 & 57 \\
\hline $\mathrm{y}$ & 26 May 1997 & 9 & 57 & 26 May 1997 & 16 & 52 & 27 May 1997 & 7 & 6 \\
\hline $\mathrm{n}$ & 07 June 1997 & 23 & 22 & 08 June 1997 & 15 & 44 & 09 June 1997 & 22 & 34 \\
\hline $\mathrm{y}$ & 19 June 1997 & 0 & 31 & 19 June 1997 & 5 & 56 & 21 June 1997 & 4 & 46 \\
\hline y & 15 July 1997 & 3 & 12 & 15 July 1997 & 6 & 31 & 16 July 1997 & 10 & 43 \\
\hline $\mathrm{n}$ & 03 August 1997 & 10 & 12 & 03 August 1997 & 14 & 29 & 04 August 1997 & 2 & 15 \\
\hline $\mathrm{n}$ & 17 August 1997 & 4 & 19 & 17 August 1997 & 6 & 2 & 18 August 1997 & 19 & 45 \\
\hline $\mathrm{y}$ & 03 September 1997 & 8 & 54 & 03 September 1997 & 13 & 55 & 03 September 1997 & 20 & 52 \\
\hline $\mathrm{n}$ & 18 September 1997 & 0 & 47 & 18 September 1997 & 4 & 20 & 20 September 1997 & 11 & 54 \\
\hline $\mathrm{n}$ & 21 September 1997 & 14 & 55 & 21 September 1997 & 22 & 24 & 22 September 1997 & 17 & 45 \\
\hline $\mathrm{n}$ & 30 September 1997 & 23 & 37 & 01 October 1997 & 17 & 0 & 02 October 1997 & 23 & 11 \\
\hline $\mathrm{y}$ & 10 October 1997 & 16 & 10 & 10 October 1997 & 22 & 17 & 12 October 1997 & 2 & 53 \\
\hline $\mathrm{n}$ & 26 October 1997 & 12 & 52 & 27 October 1997 & 10 & 41 & 28 October 1997 & 5 & 21 \\
\hline $\mathrm{y}$ & 06 November 1997 & 22 & 46 & 07 November 1997 & 15 & 50 & 08 November 1997 & 20 & 6 \\
\hline $\mathrm{y}$ & 22 November 1997 & 9 & 50 & 22 November 1997 & 19 & 22 & 23 November 1997 & 13 & 10 \\
\hline $\mathrm{y}$ & 10 December 1997 & 5 & 31 & 10 December 1997 & 19 & 37 & 11 December 1997 & 19 & 48 \\
\hline $\mathrm{y}$ & 30 December 1997 & 2 & 16 & 30 December 1997 & 9 & 27 & 31 December 1997 & 9 & 23 \\
\hline $\mathrm{y}$ & 06 January 1998 & 14 & 16 & 07 January 1998 & 3 & 54 & 08 January 1998 & 8 & 27 \\
\hline $\mathrm{n}$ & 20 January 1998 & 22 & 59 & 21 January 1998 & 6 & 16 & 22 January 1998 & 14 & 33 \\
\hline $\mathrm{y}$ & 28 January 1998 & 16 & 40 & 29 January 1998 & 21 & 28 & 31 January 1998 & 0 & 22 \\
\hline $\mathrm{n}$ & 04 February 1998 & 1 & 7 & 04 February 1998 & 5 & 49 & 05 February 1998 & 4 & 30 \\
\hline $\mathrm{y}$ & 17 February 1998 & 5 & 34 & 17 February 1998 & 11 & 34 & 18 February 1998 & 4 & 41 \\
\hline$n$ & 18 February 1998 & 8 & 42 & 19 February 1998 & 0 & 31 & 20 February 1998 & 1 & 9 \\
\hline $\mathrm{y}$ & 04 March 1998 & 12 & 0 & 04 March 1998 & 15 & 37 & 06 March 1998 & 6 & 49 \\
\hline $\mathrm{n}$ & 25 March 1998 & 10 & 17 & 25 March 1998 & 14 & 10 & 26 March 1998 & 9 & 56 \\
\hline $\mathrm{n}$ & 30 March 1998 & 23 & 26 & 31 March 1998 & 13 & 16 & 01 April 1998 & 23 & 56 \\
\hline $\mathrm{y}$ & 07 April 1998 & 17 & 38 & 08 April 1998 & 1 & 27 & 09 April 1998 & 4 & 53 \\
\hline $\mathrm{n}$ & 11 April 1998 & 5 & 8 & 11 April 1998 & 20 & 55 & 13 April 1998 & 4 & 51 \\
\hline
\end{tabular}


Table 6 (Continued)

\begin{tabular}{|c|c|c|c|c|c|c|c|c|c|}
\hline \multirow{2}{*}{$\begin{array}{l}\text { Driven } \\
\text { shock } \\
\mathrm{y}(\mathrm{yes}) \text { or } \\
\mathrm{n}(\mathrm{no})\end{array}$} & \multicolumn{3}{|l|}{ Start of the sheath } & \multicolumn{3}{|l|}{ Start of the ICME } & \multicolumn{3}{|l|}{ End of the ICME } \\
\hline & day & hour & $\min$ & day & hour & $\min$ & day & hour & $\min$ \\
\hline $\mathrm{y}$ & 01 May 1998 & 21 & 58 & 02 May 1998 & 5 & 12 & 03 May 1998 & 17 & 56 \\
\hline $\mathrm{y}$ & 03 May 1998 & 17 & 57 & 03 May 1998 & 19 & 48 & 04 May 1998 & 2 & 4 \\
\hline $\mathrm{y}$ & 04 May 1998 & 3 & 0 & 05 May 1998 & 7 & 55 & 07 May 1998 & 21 & 6 \\
\hline $\mathrm{n}$ & 02 June 1998 & 8 & 57 & 02 June 1998 & 11 & 12 & 02 June 1998 & 19 & 29 \\
\hline $\mathrm{y}$ & 13 June 1998 & 19 & 48 & 14 June 1998 & 14 & 32 & 15 June 1998 & 3 & 45 \\
\hline $\mathrm{n}$ & 24 June 1998 & 9 & 7 & 24 June 1998 & 19 & 44 & 25 June 1998 & 16 & 48 \\
\hline $\mathrm{y}$ & 25 June 1998 & 16 & 48 & 25 June 1998 & 18 & 38 & 26 June 1998 & 19 & 37 \\
\hline $\mathrm{n}$ & 05 July 1998 & 1 & 28 & O6 July 1998 & 21 & 50 & 08 July 1998 & 18 & 3 \\
\hline $\mathrm{n}$ & 10 July 1998 & 20 & 21 & 11 July 1998 & 1 & 26 & 13 July 1998 & 7 & 31 \\
\hline $\mathrm{y}$ & 31 July 1998 & 10 & 9 & 31 July 1998 & 18 & 50 & 01 August 1998 & 13 & 18 \\
\hline $\mathrm{n}$ & 01 August 1998 & 13 & 19 & 01 August 1998 & 19 & 1 & 03 August 1998 & 1 & 13 \\
\hline $\mathrm{y}$ & 10 August 1998 & 0 & 54 & 10 August 1998 & 10 & 58 & 10 August 1998 & 22 & 46 \\
\hline $\mathrm{n}$ & 11 August 1998 & 23 & 8 & 12 August 1998 & 0 & 59 & 13 August 1998 & 14 & 28 \\
\hline $\mathrm{y}$ & 19 August 1998 & 18 & 36 & 20 August 1998 & 9 & 29 & 21 August 1998 & 20 & 51 \\
\hline $\mathrm{y}$ & 26 August 1998 & 6 & 54 & 26 August 1998 & 22 & 18 & 28 August 1998 & 1 & 17 \\
\hline $\mathrm{n}$ & 22 September 1998 & 19 & 59 & 23 September 1998 & 6 & 57 & 23 September 1998 & 18 & 40 \\
\hline $\mathrm{y}$ & 24 September 1998 & 23 & 51 & 25 September 1998 & 6 & 48 & 26 September 1998 & 16 & 54 \\
\hline $\mathrm{y}$ & 18 October 1998 & 19 & 47 & 19 October 1998 & 4 & 46 & 20 October 1998 & 6 & 19 \\
\hline $\mathrm{y}$ & 23 October 1998 & 13 & 12 & 23 October 1998 & 19 & 39 & 24 October 1998 & 17 & 55 \\
\hline $\mathrm{y}$ & 07 November 1998 & 8 & 13 & 07 November 1998 & 22 & 43 & 08 November 1998 & 4 & 55 \\
\hline $\mathrm{y}$ & 08 November 1998 & 4 & 55 & 09 November 1998 & 0 & 25 & 10 November 1998 & 3 & 45 \\
\hline $\mathrm{n}$ & 13 November 1998 & 1 & 31 & 13 November 1998 & 5 & 20 & 14 November 1998 & 14 & 43 \\
\hline $\mathrm{y}$ & 30 November 1998 & 5 & 12 & 30 November 1998 & 10 & 10 & 01 December 1998 & 3 & 5 \\
\hline $\mathrm{y}$ & 26 December 1998 & 10 & 4 & 26 December 1998 & 12 & 12 & 27 December 1998 & 1 & 10 \\
\hline $\mathrm{y}$ & 28 December 1998 & 18 & 38 & 29 December 1998 & 6 & 51 & 31 December 1998 & 1 & 59 \\
\hline $\mathrm{n}$ & 04 January 1999 & 1 & 10 & 04 January 1999 & 8 & 47 & 04 January 1999 & 23 & 26 \\
\hline $\mathrm{y}$ & 13 January 1999 & 10 & 33 & 13 January 1999 & 16 & 5 & 13 January 1999 & 23 & 20 \\
\hline $\mathrm{y}$ & 22 January 1999 & 20 & 33 & 23 January 1999 & 5 & 39 & 23 January 1999 & 14 & 57 \\
\hline $\mathrm{n}$ & 13 February 1999 & 10 & 59 & 13 February 1999 & 19 & 21 & 14 February 1999 & 15 & 21 \\
\hline $\mathrm{y}$ & 17 February 1999 & 7 & 15 & 17 February 1999 & 15 & 12 & 18 February 1999 & 0 & 20 \\
\hline $\mathrm{y}$ & 18 February 1999 & 2 & 49 & 18 February 1999 & 15 & 57 & 19 February 1999 & 11 & 28 \\
\hline $\mathrm{y}$ & 10 March 1999 & 1 & 36 & 10 March 1999 & 17 & 59 & 12 March 1999 & 2 & 14 \\
\hline $\mathrm{n}$ & 19 March 1999 & 10 & 43 & 19 March 1999 & 12 & 40 & 20 March 1999 & 12 & 46 \\
\hline $\mathrm{y}$ & 16 April 1999 & 11 & 23 & 16 April 1999 & 19 & 56 & 17 April 1999 & 19 & 53 \\
\hline $\mathrm{y}$ & 20 April 1999 & 16 & 32 & 21 April 1999 & 5 & 15 & 22 April 1999 & 15 & 8 \\
\hline $\mathrm{n}$ & 15 May 1999 & 10 & 38 & 16 May 1999 & 12 & 49 & 18 May 1999 & 0 & 38 \\
\hline $\mathrm{n}$ & 28 May 1999 & 16 & 59 & 28 May 1999 & 22 & 48 & 30 May 1999 & 11 & 54 \\
\hline $\mathrm{n}$ & 02 June 1999 & 14 & 37 & 03 June 1999 & 1 & 14 & 03 June 1999 & 22 & 57 \\
\hline $\mathrm{n}$ & 26 June 1999 & 3 & 30 & 26 June 1999 & 6 & 51 & 26 June 1999 & 20 & 14 \\
\hline $\mathrm{y}$ & 02 July 1999 & 1 & 4 & 03 July 1999 & 7 & 28 & 05 July 1999 & 14 & 52 \\
\hline $\mathrm{y}$ & 06 July 1999 & 15 & 10 & 06 July 1999 & 21 & 26 & 07 July 1999 & 5 & 53 \\
\hline
\end{tabular}


Table 6 (Continued)

\begin{tabular}{|c|c|c|c|c|c|c|c|c|c|}
\hline \multirow{2}{*}{$\begin{array}{l}\text { Driven } \\
\text { shock } \\
\mathrm{y}(\text { yes) or } \\
\mathrm{n} \text { (no) }\end{array}$} & \multicolumn{3}{|l|}{ Start of the sheath } & \multicolumn{3}{|l|}{ Start of the ICME } & \multicolumn{3}{|l|}{ End of the ICME } \\
\hline & day & hour & $\min$ & day & hour & $\min$ & day & hour & $\min$ \\
\hline $\mathrm{n}$ & 07 July 1999 & 5 & 54 & 07 July 1999 & 6 & 48 & 08 July 1999 & 2 & 5 \\
\hline $\mathrm{y}$ & 08 July 1999 & 4 & 31 & 08 July 1999 & 8 & 6 & 09 July 1999 & 2 & 59 \\
\hline $\mathrm{y}$ & 12 July 1999 & 2 & 24 & 12 July 1999 & 12 & 53 & 13 July 1999 & 14 & 39 \\
\hline $\mathrm{y}$ & 27 July 1999 & 0 & 28 & 27 July 1999 & 16 & 38 & 29 July 1999 & 11 & 5 \\
\hline $\mathrm{n}$ & 30 July 1999 & 11 & 4 & 30 July 1999 & 23 & 56 & 31 July 1999 & 18 & 25 \\
\hline $\mathrm{n}$ & 31 July 1999 & 23 & 27 & 01 August 1999 & 4 & 37 & 02 August 1999 & 4 & 56 \\
\hline y & 04 August 1999 & 2 & 19 & 04 August 1999 & 9 & 11 & 06 August 1999 & 2 & 15 \\
\hline $\mathrm{y}$ & 08 August 1999 & 18 & 40 & 08 August 1999 & 23 & 13 & 10 August 1999 & 15 & 37 \\
\hline $\mathrm{n}$ & 21 August 1999 & 7 & 0 & 21 August 1999 & 15 & 20 & 23 August 1999 & 7 & 27 \\
\hline $\mathrm{y}$ & 15 September 1999 & 8 & 1 & 15 September 1999 & 13 & 24 & 15 September 1999 & 20 & 25 \\
\hline y & 15 September 1999 & 20 & 25 & 16 September 1999 & 2 & 1 & 16 September 1999 & 20 & 21 \\
\hline $\mathrm{n}$ & 21 September 1999 & 7 & 10 & 21 September 1999 & 13 & 9 & 22 September 1999 & 11 & 53 \\
\hline $\mathrm{y}$ & 22 September 1999 & 12 & 22 & 22 September 1999 & 19 & 51 & 23 September 1999 & 9 & 38 \\
\hline $\mathrm{y}$ & 21 October 1999 & 2 & 22 & 21 October 1999 & 9 & 36 & 22 October 1999 & 6 & 35 \\
\hline$n$ & 05 November 1999 & 20 & 12 & 06 November 1999 & 0 & 53 & 06 November 1999 & 9 & 23 \\
\hline $\mathrm{n}$ & 12 November 1999 & 12 & 43 & 12 November 1999 & 19 & 10 & 13 November 1999 & 12 & 50 \\
\hline $\mathrm{y}$ & 13 November 1999 & 12 & 50 & 14 November 1999 & 1 & 29 & 14 November 1999 & 8 & 14 \\
\hline $\mathrm{n}$ & 22 November 1999 & 0 & 17 & 22 November 1999 & 11 & 57 & 24 November 1999 & 6 & 21 \\
\hline $\mathrm{y}$ & 30 November 1999 & 6 & 44 & 30 November 1999 & 13 & 57 & 02 December 1999 & 18 & 17 \\
\hline$n$ & 11 December 1999 & 12 & 53 & 12 December 1999 & 1 & 57 & 12 December 1999 & 15 & 58 \\
\hline $\mathrm{y}$ & 12 December 1999 & 15 & 58 & 12 December 1999 & 20 & 17 & 13 December 1999 & 17 & 18 \\
\hline $\mathrm{n}$ & 14 December 1999 & 1 & 26 & 14 December 1999 & 5 & 4 & 14 December 1999 & 19 & 54 \\
\hline $\mathrm{y}$ & 26 December 1999 & 22 & 28 & 27 December 1999 & 18 & 2 & 28 December 1999 & 5 & 21 \\
\hline $\mathrm{y}$ & 22 January 2000 & 2 & 11 & 22 January 2000 & 17 & 51 & 25 January 2000 & 5 & 10 \\
\hline $\mathrm{y}$ & 11 February 2000 & 2 & 52 & 11 February 2000 & 8 & 39 & 11 February 2000 & 22 & 7 \\
\hline $\mathrm{y}$ & 11 February 2000 & 23 & 53 & 12 February 2000 & 6 & 21 & 13 February 2000 & 0 & 53 \\
\hline $\mathrm{y}$ & 14 February 2000 & 7 & 36 & 14 February 2000 & 13 & 34 & 15 February 2000 & 16 & 6 \\
\hline $\mathrm{y}$ & 20 February 2000 & 21 & 43 & 21 February 2000 & 6 & 14 & 22 February 2000 & 12 & 51 \\
\hline $\mathrm{n}$ & 01 March 2000 & 2 & 25 & 01 March 2000 & 3 & 58 & 02 March 2000 & 3 & 27 \\
\hline $\mathrm{n}$ & 29 March 2000 & 6 & 46 & 29 March 2000 & 22 & 42 & 01 April 2000 & 6 & 57 \\
\hline $\mathrm{n}$ & 03 April 2000 & 16 & 3 & 04 April 2000 & 2 & 34 & 06 April 2000 & 1 & 56 \\
\hline $\mathrm{y}$ & 06 April 2000 & 16 & 45 & 07 April 2000 & 9 & 12 & 08 April 2000 & 6 & 8 \\
\hline $\mathrm{n}$ & 18 April 2000 & 20 & 46 & 19 April 2000 & 5 & 39 & 19 April 2000 & 12 & 29 \\
\hline $\mathrm{n}$ & 24 April 2000 & 4 & 24 & 24 April 2000 & 9 & 32 & 24 April 2000 & 13 & 50 \\
\hline $\mathrm{y}$ & 02 May 2000 & 9 & 32 & 02 May 2000 & 22 & 49 & 04 May 2000 & 7 & 38 \\
\hline $\mathrm{n}$ & 13 May 2000 & 17 & 27 & 14 May 2000 & 2 & 24 & 14 May 2000 & 20 & 5 \\
\hline $\mathrm{n}$ & 15 May 2000 & 9 & 24 & 15 May 2000 & 14 & 31 & 16 May 2000 & 5 & 25 \\
\hline y & 23 Мау 2000 & 17 & 25 & 24 May 2000 & 12 & 18 & 25 May 2000 & 9 & 4 \\
\hline $\mathrm{y}$ & 04 June 2000 & 14 & 56 & 05 June 2000 & 0 & 45 & 06 June 2000 & 16 & 13 \\
\hline $\mathrm{y}$ & 08 June 2000 & 9 & 0 & 09 June 2000 & 1 & 39 & 10 June 2000 & 0 & 49 \\
\hline $\mathrm{n}$ & 12 June 2000 & 18 & 33 & 13 June 2000 & 13 & 9 & 14 June 2000 & 7 & 0 \\
\hline
\end{tabular}


Table 6 (Continued)

\begin{tabular}{|c|c|c|c|c|c|c|c|c|c|}
\hline \multirow{2}{*}{$\begin{array}{l}\text { Driven } \\
\text { shock } \\
\mathrm{y}(\text { yes }) \text { or } \\
\mathrm{n}(\text { no })\end{array}$} & \multicolumn{3}{|l|}{ Start of the sheath } & \multicolumn{3}{|l|}{ Start of the ICME } & \multicolumn{3}{|l|}{ End of the ICME } \\
\hline & day & hour & $\min$ & day & hour & $\min$ & day & hour & $\min$ \\
\hline $\mathrm{y}$ & 23 June 2000 & 12 & 46 & 24 June 2000 & 7 & 38 & 25 June 2000 & 20 & 32 \\
\hline $\mathrm{n}$ & 26 June 2000 & 0 & 19 & 26 June 2000 & 10 & 48 & 27 June 2000 & 0 & 31 \\
\hline $\mathrm{n}$ & 30 June 2000 & 23 & 55 & 01 July 2000 & 8 & 34 & 02 July 2000 & 6 & 32 \\
\hline $\mathrm{y}$ & 10 July 2000 & 6 & 49 & 11 July 2000 & 2 & 59 & 11 July 2000 & 12 & 6 \\
\hline $\mathrm{y}$ & 11 July 2000 & 12 & 16 & 11 July 2000 & 23 & 39 & 13 July 2000 & 3 & 19 \\
\hline $\mathrm{y}$ & 13 July 2000 & 9 & 52 & 13 July 2000 & 13 & 9 & 14 July 2000 & 5 & 35 \\
\hline $\mathrm{y}$ & 19 July 2000 & 15 & 29 & 20 July 2000 & 9 & 58 & 21 July 2000 & 6 & 42 \\
\hline $\mathrm{n}$ & 23 July 2000 & 15 & 9 & 23 July 2000 & 20 & 23 & 26 July 2000 & 3 & 50 \\
\hline $\mathrm{n}$ & 26 July 2000 & 4 & 0 & 26 July 2000 & 4 & 20 & 26 July 2000 & 17 & 40 \\
\hline $\mathrm{y}$ & 26 July 2000 & 19 & 7 & 27 July 2000 & 9 & 28 & 27 July 2000 & 20 & 56 \\
\hline $\mathrm{y}$ & 28 July 2000 & 6 & 29 & 28 July 2000 & 13 & 5 & 29 July 2000 & 10 & 33 \\
\hline $\mathrm{y}$ & 10 August 2000 & 5 & 3 & 10 August 2000 & 20 & 27 & 11 August 2000 & 16 & 9 \\
\hline $\mathrm{y}$ & 11 August 2000 & 19 & 1 & 12 August 2000 & 6 & 0 & 13 August 2000 & 22 & 53 \\
\hline $\mathrm{n}$ & 02 September 2000 & 22 & 26 & 02 September 2000 & 22 & 51 & 03 September 2000 & 13 & 26 \\
\hline $\mathrm{y}$ & 04 September 2000 & 13 & 40 & 04 September 2000 & 18 & 9 & 05 September 2000 & 10 & 25 \\
\hline $\mathrm{y}$ & 06 September 2000 & 17 & 6 & 07 September 2000 & 3 & 6 & 08 September 2000 & 7 & 1 \\
\hline $\mathrm{n}$ & 08 September 2000 & 7 & 3 & 08 September 2000 & 19 & 57 & 10 September 2000 & 11 & 10 \\
\hline y & 17 September 2000 & 16 & 8 & 18 September 2000 & 0 & 32 & 20 September 2000 & 11 & 14 \\
\hline $\mathrm{y}$ & 03 October 2000 & 0 & 54 & 03 October 2000 & 11 & 15 & 05 October 2000 & 3 & 34 \\
\hline $\mathrm{y}$ & 05 October 2000 & 3 & 35 & 05 October 2000 & 10 & 50 & 07 October 2000 & 14 & 23 \\
\hline $\mathrm{y}$ & 12 October 2000 & 22 & 31 & 13 October 2000 & 17 & 2 & 14 October 2000 & 18 & 11 \\
\hline $\mathrm{y}$ & 28 October 2000 & 10 & 0 & 28 October 2000 & 22 & 36 & 29 October 2000 & 23 & 24 \\
\hline $\mathrm{y}$ & 31 October 2000 & 17 & 20 & 01 November 2000 & 6 & 52 & 03 November 2000 & 14 & 44 \\
\hline $\mathrm{y}$ & 06 November 2000 & 9 & 52 & 06 November 2000 & 22 & 57 & 08 November 2000 & 10 & 4 \\
\hline $\mathrm{y}$ & 10 November 2000 & 6 & 21 & 10 November 2000 & 12 & 34 & 11 November 2000 & 4 & 26 \\
\hline $\mathrm{y}$ & 11 November 2000 & 4 & 28 & 11 November 2000 & 6 & 41 & 11 November 2000 & 23 & 21 \\
\hline $\mathrm{y}$ & 26 November 2000 & 6 & 0 & 27 November 2000 & 8 & 50 & 28 November 2000 & 3 & 19 \\
\hline $\mathrm{y}$ & 28 November 2000 & 5 & 30 & 28 November 2000 & 12 & 12 & 29 November 2000 & 20 & 55 \\
\hline $\mathrm{y}$ & 03 December 2000 & 4 & 10 & 03 December 2000 & 12 & 49 & 05 December 2000 & 6 & 41 \\
\hline $\mathrm{n}$ & 21 December 2000 & 12 & 49 & 22 December 2000 & 3 & 3 & 22 December 2000 & 18 & 30 \\
\hline $\mathrm{n}$ & 22 December 2000 & 18 & 40 & 23 December 2000 & 0 & 15 & 23 December 2000 & 20 & 20 \\
\hline $\mathrm{y}$ & 13 January 2001 & 2 & 32 & 13 January 2001 & 9 & 53 & 14 January 2001 & 18 & 42 \\
\hline $\mathrm{y}$ & 23 January 2001 & 10 & 57 & 24 January 2001 & 9 & 27 & 26 January 2001 & 11 & 59 \\
\hline $\mathrm{y}$ & 31 January 2001 & 8 & 15 & 01 February 2001 & 0 & 8 & 02 February 2001 & 15 & 18 \\
\hline $\mathrm{y}$ & 03 March 2001 & 11 & 24 & 04 March 2001 & 5 & 59 & 05 March 2001 & 2 & 19 \\
\hline $\mathrm{y}$ & 19 March 2001 & 11 & 17 & 19 March 2001 & 18 & 16 & 21 March 2001 & 23 & 44 \\
\hline $\mathrm{y}$ & 27 March 2001 & 1 & 53 & 27 March 2001 & 4 & 39 & 27 March 2001 & 17 & 31 \\
\hline $\mathrm{y}$ & 27 March 2001 & 17 & 53 & 28 March 2001 & 17 & 55 & 30 March 2001 & 19 & 19 \\
\hline $\mathrm{y}$ & 31 March 2001 & 1 & 0 & 31 March 2001 & 6 & 6 & 31 March 2001 & 22 & 10 \\
\hline $\mathrm{y}$ & 31 March 2001 & 22 & 11 & 01 April 2001 & 5 & 43 & 03 April 2001 & 16 & 54 \\
\hline $\mathrm{y}$ & 04 April 2001 & 15 & 0 & 04 April 2001 & 17 & 55 & 05 April 2001 & 7 & 37 \\
\hline
\end{tabular}


Table 6 (Continued)

\begin{tabular}{|c|c|c|c|c|c|c|c|c|c|}
\hline \multirow{2}{*}{$\begin{array}{l}\text { Driven } \\
\text { shock } \\
\mathrm{y}(\text { yes) or } \\
\mathrm{n} \text { (no) }\end{array}$} & \multicolumn{3}{|l|}{ Start of the sheath } & \multicolumn{3}{|l|}{ Start of the ICME } & \multicolumn{3}{|l|}{ End of the ICME } \\
\hline & day & hour & $\min$ & day & hour & $\min$ & day & hour & $\min$ \\
\hline $\mathrm{y}$ & 07 April 2001 & 17 & 34 & 07 April 2001 & 18 & 11 & 08 April 2001 & 11 & 2 \\
\hline $\mathrm{y}$ & 08 April 2001 & 11 & 3 & 08 April 2001 & 14 & 20 & 09 April 2001 & 18 & 48 \\
\hline $\mathrm{y}$ & 11 April 2001 & 13 & 43 & 12 April 2001 & 8 & 44 & 13 April 2001 & 5 & 38 \\
\hline $\mathrm{y}$ & 13 April 2001 & 7 & 30 & 13 April 2001 & 11 & 1 & 14 April 2001 & 10 & 37 \\
\hline $\mathrm{y}$ & 18 April 2001 & 0 & 50 & 18 April 2001 & 12 & 23 & 20 April 2001 & 0 & 40 \\
\hline $\mathrm{y}$ & 21 April 2001 & 16 & 18 & 22 April 2001 & 0 & 32 & 23 April 2001 & 0 & 26 \\
\hline y & 28 April 2001 & 5 & 2 & 28 April 2001 & 15 & 58 & 30 April 2001 & 0 & 43 \\
\hline $\mathrm{y}$ & 08 May 2001 & 10 & 42 & 09 May 2001 & 13 & 24 & 10 May 2001 & 21 & 59 \\
\hline $\mathrm{y}$ & 27 May 2001 & 14 & 55 & 28 May 2001 & 6 & 47 & 30 May 2001 & 11 & 16 \\
\hline $\mathrm{y}$ & 07 June 2001 & 9 & 52 & 07 June 2001 & 18 & 26 & 08 June 2001 & 8 & 28 \\
\hline$n$ & 26 June 2001 & 21 & 33 & 27 June 2001 & 3 & 43 & 28 June 2001 & 18 & 21 \\
\hline $\mathrm{n}$ & 08 July 2001 & 3 & 50 & 09 July 2001 & 3 & 45 & 11 July 2001 & 14 & 59 \\
\hline $\mathrm{y}$ & 03 August 2001 & 7 & 24 & 03 August 2001 & 12 & 20 & 03 August 2001 & 14 & 56 \\
\hline $\mathrm{y}$ & 12 August 2001 & 11 & 51 & 13 August 2001 & 1 & 14 & 13 August 2001 & 17 & 8 \\
\hline $\mathrm{y}$ & 17 August 2001 & 11 & 5 & 17 August 2001 & 20 & 56 & 18 August 2001 & 14 & 12 \\
\hline $\mathrm{y}$ & 27 August 2001 & 19 & 51 & 28 August 2001 & 3 & 55 & 28 August 2001 & 18 & 48 \\
\hline $\mathrm{y}$ & 30 August 2001 & 14 & 13 & 30 August 2001 & 18 & 48 & 31 August 2001 & 7 & 24 \\
\hline $\mathrm{n}$ & 13 September 2001 & 2 & 37 & 13 September 2001 & 4 & 26 & 13 September 2001 & 17 & 35 \\
\hline $\mathrm{y}$ & 14 September 2001 & 2 & 11 & 14 September 2001 & 2 & 45 & 14 September 2001 & 21 & 45 \\
\hline $\mathrm{n}$ & 23 September 2001 & 6 & 30 & 24 September 2001 & 1 & 37 & 24 September 2001 & 20 & 37 \\
\hline $\mathrm{y}$ & 25 September 2001 & 20 & 10 & 26 September 2001 & 1 & 13 & 26 September 2001 & 10 & 29 \\
\hline $\mathrm{y}$ & 29 September 2001 & 9 & 39 & 29 September 2001 & 12 & 8 & 30 September 2001 & 16 & 54 \\
\hline $\mathrm{y}$ & 30 September 2001 & 19 & 21 & 30 September 2001 & 22 & 54 & 01 October 2001 & 21 & 54 \\
\hline $\mathrm{y}$ & 01 October 2001 & 21 & 59 & 02 October 2001 & 4 & 52 & 02 October 2001 & 12 & 57 \\
\hline $\mathrm{n}$ & 02 October 2001 & 23 & 10 & 03 October 2001 & 0 & 40 & 03 October 2001 & 17 & 13 \\
\hline $\mathrm{n}$ & 12 October 2001 & 0 & 7 & 12 October 2001 & 3 & 59 & 12 October 2001 & 9 & 4 \\
\hline $\mathrm{y}$ & 21 October 2001 & 16 & 46 & 21 October 2001 & 21 & 25 & 22 October 2001 & 21 & 33 \\
\hline $\mathrm{y}$ & 28 October 2001 & 3 & 25 & 28 October 2001 & 11 & 46 & 31 October 2001 & 10 & 32 \\
\hline $\mathrm{y}$ & 31 October 2001 & 14 & 24 & 31 October 2001 & 21 & 40 & 02 November 2001 & 5 & 29 \\
\hline $\mathrm{y}$ & 19 November 2001 & 18 & 11 & 20 November 2001 & 6 & 45 & 21 November 2001 & 15 & 29 \\
\hline $\mathrm{y}$ & 24 November 2001 & 6 & 0 & 24 November 2001 & 17 & 1 & 25 November 2001 & 16 & 37 \\
\hline $\mathrm{y}$ & 29 December 2001 & 5 & 22 & 29 December 2001 & 23 & 8 & 30 December 2001 & 19 & 3 \\
\hline $\mathrm{y}$ & 30 December 2001 & 20 & 8 & 30 December 2001 & 23 & 9 & 31 December 2001 & 14 & 59 \\
\hline $\mathrm{y}$ & 31 January 2002 & 21 & 36 & 01 February 2002 & 12 & 22 & 02 February 2002 & 8 & 32 \\
\hline $\mathrm{y}$ & 28 February 2002 & 5 & 3 & 28 February 2002 & 17 & 32 & 01 March 2002 & 10 & 58 \\
\hline $\mathrm{y}$ & 18 March 2002 & 13 & 27 & 19 March 2002 & 5 & 40 & 20 March 2002 & 9 & 59 \\
\hline $\mathrm{n}$ & 20 March 2002 & 13 & 37 & 21 March 2002 & 9 & 1 & 22 March 2002 & 4 & 10 \\
\hline y & 23 March 2002 & 11 & 40 & 24 March 2002 & 12 & 28 & 25 March 2002 & 21 & 19 \\
\hline $\mathrm{n}$ & 11 April 2002 & 22 & 3 & 12 April 2002 & 0 & 36 & 13 April 2002 & 15 & 14 \\
\hline $\mathrm{n}$ & 14 April 2002 & 10 & 25 & 14 April 2002 & 14 & 34 & 16 April 2002 & 6 & 5 \\
\hline $\mathrm{y}$ & 17 April 2002 & 11 & 12 & 17 April 2002 & 19 & 12 & 19 April 2002 & 2 & 41 \\
\hline
\end{tabular}


Table 6 (Continued)

\begin{tabular}{|c|c|c|c|c|c|c|c|c|c|}
\hline \multirow{2}{*}{$\begin{array}{l}\text { Driven } \\
\text { shock } \\
\mathrm{y}(\text { yes }) \text { or } \\
\mathrm{n}(\text { no })\end{array}$} & \multicolumn{3}{|l|}{ Start of the sheath } & \multicolumn{3}{|l|}{ Start of the ICME } & \multicolumn{3}{|l|}{ End of the ICME } \\
\hline & day & hour & $\min$ & day & hour & $\min$ & day & hour & $\min$ \\
\hline $\mathrm{y}$ & 19 April 2002 & 8 & 37 & 20 April 2002 & 0 & 48 & 21 April 2002 & 16 & 24 \\
\hline $\mathrm{y}$ & 23 April 2002 & 4 & 54 & 23 April 2002 & 8 & 53 & 24 April 2002 & 16 & 39 \\
\hline $\mathrm{y}$ & 10 May 2002 & 11 & 34 & 10 May 2002 & 23 & 23 & 11 May 2002 & 9 & 48 \\
\hline $\mathrm{y}$ & 11 May 2002 & 9 & 51 & 11 May 2002 & 12 & 5 & 12 May 2002 & 1 & 48 \\
\hline $\mathrm{y}$ & 18 May 2002 & 20 & 15 & 19 May 2002 & 3 & 38 & 20 May 2002 & 0 & 57 \\
\hline $\mathrm{y}$ & 20 May 2002 & 3 & 41 & 20 May 2002 & 10 & 26 & 21 May 2002 & 14 & 47 \\
\hline $\mathrm{y}$ & 21 May 2002 & 22 & 5 & 21 May 2002 & 23 & 21 & 22 May 2002 & 8 & 42 \\
\hline $\mathrm{y}$ & 23 May 2002 & 10 & 57 & 23 May 2002 & 15 & 45 & 25 May 2002 & 15 & 35 \\
\hline $\mathrm{y}$ & 30 May 2002 & 2 & 4 & 30 May 2002 & 7 & 24 & 31 May 2002 & 3 & 46 \\
\hline $\mathrm{y}$ & 17 July 2002 & 16 & 6 & 18 July 2002 & 9 & 37 & 19 July 2002 & 8 & 47 \\
\hline $\mathrm{y}$ & 19 July 2002 & 10 & 17 & 20 July 2002 & 1 & 21 & 21 July 2002 & 12 & 10 \\
\hline $\mathrm{y}$ & 01 August 2002 & 5 & 16 & 01 August 2002 & 9 & 58 & 01 August 2002 & 23 & 11 \\
\hline $\mathrm{y}$ & 01 August 2002 & 23 & 11 & 02 August 2002 & 7 & 3 & 03 August 2002 & 7 & 58 \\
\hline $\mathrm{y}$ & 18 August 2002 & 18 & 31 & 19 August 2002 & 15 & 32 & 21 August 2002 & 15 & 5 \\
\hline $\mathrm{y}$ & 26 August 2002 & 11 & 30 & 26 August 2002 & 14 & 44 & 27 August 2002 & 11 & 1 \\
\hline $\mathrm{n}$ & 29 August 2002 & 8 & 42 & 29 August 2002 & 14 & 10 & 30 August 2002 & 9 & 13 \\
\hline $\mathrm{y}$ & 07 September 2002 & 16 & 49 & 08 September 2002 & 4 & 49 & 08 September 2002 & 19 & 27 \\
\hline $\mathrm{n}$ & 08 September 2002 & 19 & 27 & 09 September 2002 & 0 & 15 & 10 September 2002 & 21 & 46 \\
\hline $\mathrm{n}$ & 19 September 2002 & 6 & 18 & 19 September 2002 & 19 & 51 & 20 September 2002 & 22 & 44 \\
\hline $\mathrm{y}$ & 30 September 2002 & 8 & 17 & 30 September 2002 & 21 & 49 & 01 October 2002 & 14 & 58 \\
\hline $\mathrm{y}$ & 02 October 2002 & 22 & 59 & 03 October 2002 & 18 & 0 & 04 October 2002 & 19 & 23 \\
\hline $\mathrm{y}$ & 16 November 2002 & 23 & 47 & 17 November 2002 & 18 & 50 & 19 November 2002 & 0 & 50 \\
\hline $\mathrm{n}$ & 20 December 2002 & 18 & 0 & 21 December 2002 & 8 & 27 & 22 December 2002 & 8 & 31 \\
\hline $\mathrm{n}$ & 26 January 2003 & 21 & 54 & 27 January 2003 & 2 & 33 & 27 January 2003 & 16 & 51 \\
\hline $\mathrm{y}$ & 01 February 2003 & 13 & 43 & 01 February 2003 & 20 & 32 & 03 February 2003 & 2 & 29 \\
\hline $\mathrm{n}$ & 17 February 2003 & 22 & 21 & 18 February 2003 & 4 & 41 & 19 February 2003 & 11 & 34 \\
\hline $\mathrm{y}$ & 20 March 2003 & 4 & 46 & 20 March 2003 & 12 & 56 & 20 March 2003 & 23 & 8 \\
\hline $\mathrm{y}$ & 09 May 2003 & 5 & 26 & 09 May 2003 & 8 & 25 & 10 May 2003 & 12 & 27 \\
\hline $\mathrm{y}$ & 29 May 2003 & 12 & 24 & 29 May 2003 & 13 & 40 & 29 May 2003 & 19 & 2 \\
\hline $\mathrm{y}$ & 29 May 2003 & 19 & 2 & 30 May 2003 & 1 & 53 & 30 May 2003 & 15 & 6 \\
\hline $\mathrm{y}$ & 30 May 2003 & 16 & 23 & 30 May 2003 & 22 & 54 & 31 May 2003 & 16 & 43 \\
\hline $\mathrm{n}$ & 15 June 2003 & 14 & 44 & 15 June 2003 & 20 & 42 & 16 June 2003 & 21 & 16 \\
\hline $\mathrm{n}$ & 16 June 2003 & 23 & 6 & 17 June 2003 & 1 & 28 & 18 June 2003 & 5 & 19 \\
\hline $\mathrm{y}$ & 06 July 2003 & 13 & 4 & 06 July 2003 & 20 & 31 & 07 July 2003 & 12 & 16 \\
\hline $\mathrm{n}$ & 23 July 2003 & 14 & 36 & 23 July 2003 & 16 & 42 & 24 July 2003 & 14 & 58 \\
\hline $\mathrm{n}$ & 04 August 2003 & 18 & 2 & 05 August 2003 & 0 & 44 & 06 August 2003 & 1 & 56 \\
\hline $\mathrm{n}$ & 15 August 2003 & 12 & 37 & 16 August 2003 & 2 & 31 & 17 August 2003 & 14 & 22 \\
\hline $\mathrm{y}$ & 17 August 2003 & 14 & 22 & 18 August 2003 & 2 & 45 & 19 August 2003 & 17 & 17 \\
\hline $\mathrm{n}$ & 21 October 2003 & 20 & 17 & 22 October 2003 & 15 & 43 & 24 October 2003 & 9 & 8 \\
\hline $\mathrm{y}$ & 24 October 2003 & 15 & 18 & 24 October 2003 & 19 & 22 & 25 October 2003 & 12 & 2 \\
\hline $\mathrm{n}$ & 25 October 2003 & 12 & 34 & 25 October 2003 & 14 & 41 & 26 October 2003 & 5 & 36 \\
\hline
\end{tabular}


Table 6 (Continued)

\begin{tabular}{|c|c|c|c|c|c|c|c|c|c|}
\hline \multirow{2}{*}{$\begin{array}{l}\text { Driven } \\
\text { shock } \\
\text { y(yes) or } \\
\text { n(no) }\end{array}$} & \multicolumn{3}{|l|}{ Start of the sheath } & \multicolumn{3}{|l|}{ Start of the ICME } & \multicolumn{3}{|l|}{ End of the ICME } \\
\hline & day & hour & $\min$ & day & hour & $\min$ & day & hour & $\min$ \\
\hline $\mathrm{y}$ & 26 October 2003 & 8 & 53 & 26 October 2003 & 10 & 5 & 26 October 2003 & 19 & 14 \\
\hline $\mathrm{y}$ & 26 October 2003 & 19 & 14 & 26 October 2003 & 21 & 13 & 28 October 2003 & 2 & 13 \\
\hline $\mathrm{y}$ & 04 November 2003 & 6 & 32 & 04 November 2003 & 7 & 45 & 04 November 2003 & 15 & 57 \\
\hline $\mathrm{y}$ & 06 November 2003 & 19 & 29 & 07 November 2003 & 14 & 57 & 08 November 2003 & 3 & 57 \\
\hline $\mathrm{y}$ & 15 November 2003 & 5 & 54 & 15 November 2003 & 14 & 59 & 16 November 2003 & 9 & 39 \\
\hline $\mathrm{y}$ & 20 November 2003 & 8 & 5 & 20 November 2003 & 11 & 57 & 21 November 2003 & 4 & 34 \\
\hline $\mathrm{y}$ & 09 January 2004 & 15 & 26 & 10 January 2004 & 4 & 12 & 11 January 2004 & 3 & 12 \\
\hline y & 22 January 2004 & 1 & 38 & 22 January 2004 & 10 & 35 & 23 January 2004 & 6 & 57 \\
\hline $\mathrm{y}$ & 23 January 2004 & 14 & 57 & 23 January 2004 & 23 & 32 & 25 January 2004 & 3 & 55 \\
\hline$n$ & 26 January 2004 & 19 & 5 & 26 January 2004 & 21 & 33 & 27 January 2004 & 7 & 1 \\
\hline $\mathrm{y}$ & 03 April 2004 & 9 & 51 & 04 April 2004 & 0 & 14 & 05 April 2004 & 19 & 18 \\
\hline y & 09 April 2004 & 2 & 33 & 09 April 2004 & 16 & 58 & 10 April 2004 & 20 & 15 \\
\hline $\mathrm{y}$ & 10 April 2004 & 20 & 15 & 11 April 2004 & 4 & 34 & 11 April 2004 & 23 & 36 \\
\hline $\mathrm{n}$ & 12 April 2004 & 5 & 14 & 12 April 2004 & 8 & 10 & 12 April 2004 & 18 & 26 \\
\hline $\mathrm{y}$ & 26 April 2004 & 16 & 4 & 26 April 2004 & 18 & 7 & 27 April 2004 & 10 & 28 \\
\hline $\mathrm{n}$ & 29 April 2004 & 19 & 17 & 30 April 2004 & 1 & 8 & 01 May 2004 & 12 & 21 \\
\hline $\mathrm{n}$ & 01 May 2004 & 12 & 21 & 01 May 2004 & 16 & 14 & 02 May 2004 & 22 & 9 \\
\hline $\mathrm{n}$ & 07 June 2004 & 4 & 35 & 07 June 2004 & 9 & 1 & 08 June 2004 & 4 & 24 \\
\hline y & 22 July 2004 & 10 & 40 & 22 July 2004 & 18 & 19 & 24 July 2004 & 1 & 24 \\
\hline $\mathrm{y}$ & 24 July 2004 & 6 & 13 & 24 July 2004 & 12 & 40 & 25 July 2004 & 16 & 15 \\
\hline$n$ & 25 July 2004 & 16 & 15 & 25 July 2004 & 21 & 42 & 26 July 2004 & 18 & 14 \\
\hline $\mathrm{y}$ & 26 July 2004 & 22 & 54 & 27 July 2004 & 2 & 59 & 27 July 2004 & 15 & 58 \\
\hline y & 30 July 2004 & 21 & 14 & 31 July 2004 & 4 & 44 & 01 August 2004 & 1 & 51 \\
\hline y & 01 August 2004 & 2 & 26 & 01 August 2004 & 11 & 49 & 02 August 2004 & 19 & 4 \\
\hline $\mathrm{y}$ & 29 August 2004 & 10 & 9 & 29 August 2004 & 20 & 8 & 30 August 2004 & 22 & 32 \\
\hline $\mathrm{y}$ & 13 September 2004 & 20 & 9 & 14 September 2004 & 0 & 40 & 14 September 2004 & 18 & 33 \\
\hline $\mathrm{n}$ & 18 September 2004 & 10 & 22 & 18 September 2004 & 13 & 45 & 20 September 2004 & 8 & 46 \\
\hline $\mathrm{y}$ & 22 September 2004 & 6 & 54 & 22 September 2004 & 13 & 16 & 23 September 2004 & 5 & 14 \\
\hline $\mathrm{y}$ & 07 November 2004 & 2 & 56 & 07 November 2004 & 4 & 27 & 07 November 2004 & 9 & 54 \\
\hline y & 07 November 2004 & 18 & 34 & 07 November 2004 & 23 & 2 & 09 November 2004 & 9 & 23 \\
\hline $\mathrm{y}$ & 09 November 2004 & 9 & 23 & 09 November 2004 & 12 & 2 & 09 November 2004 & 18 & 56 \\
\hline y & 09 November 2004 & 18 & 56 & 09 November 2004 & 20 & 59 & 10 November 2004 & 17 & 14 \\
\hline y & 11 November 2004 & 17 & 1 & 12 November 2004 & 9 & 25 & 13 November 2004 & 7 & 15 \\
\hline $\mathrm{y}$ & 11 December 2004 & 13 & 50 & 12 December 2004 & 23 & 32 & 13 December 2004 & 20 & 17 \\
\hline $\mathrm{n}$ & 27 December 2004 & 3 & 6 & 27 December 2004 & 17 & 16 & 29 December 2004 & 4 & 27 \\
\hline $\mathrm{y}$ & 07 January 2005 & 9 & 29 & 07 January 2005 & 15 & 51 & 08 January 2005 & 15 & 20 \\
\hline $\mathrm{n}$ & 08 January 2005 & 16 & 20 & 09 January 2005 & 0 & 39 & 09 January 2005 & 18 & 39 \\
\hline $\mathrm{n}$ & 16 January 2005 & 9 & 52 & 16 January 2005 & 14 & 34 & 17 January 2005 & 7 & 30 \\
\hline $\mathrm{n}$ & 17 January 2005 & 7 & 30 & 17 January 2005 & 14 & 11 & 18 January 2005 & 4 & 12 \\
\hline $\mathrm{y}$ & 18 January 2005 & 18 & 58 & 18 January 2005 & 23 & 7 & 20 January 2005 & 2 & 15 \\
\hline $\mathrm{y}$ & 21 January 2005 & 17 & 13 & 21 January 2005 & 18 & 47 & 22 January 2005 & 21 & 8 \\
\hline
\end{tabular}


Table 6 (Continued)

\begin{tabular}{|c|c|c|c|c|c|c|c|c|c|}
\hline \multirow{2}{*}{$\begin{array}{l}\text { Driven } \\
\text { shock } \\
\mathrm{y}(\text { yes }) \text { or } \\
\mathrm{n}(\text { no })\end{array}$} & \multicolumn{3}{|l|}{ Start of the sheath } & \multicolumn{3}{|l|}{ Start of the ICME } & \multicolumn{3}{|l|}{ End of the ICME } \\
\hline & day & hour & $\min$ & day & hour & $\min$ & day & hour & $\min$ \\
\hline $\mathrm{n}$ & 31 January 2005 & 8 & 54 & 31 January 2005 & 12 & 9 & 02 February 2005 & 10 & 6 \\
\hline $\mathrm{n}$ & 17 February 2005 & 21 & 29 & 18 February 2005 & 15 & 47 & 19 February 2005 & 5 & 50 \\
\hline $\mathrm{n}$ & 19 February 2005 & 18 & 51 & 20 February 2005 & 0 & 53 & 21 February 2005 & 17 & 29 \\
\hline $\mathrm{n}$ & 22 February 2005 & 11 & 49 & 22 February 2005 & 17 & 24 & 23 February 2005 & 19 & 36 \\
\hline $\mathrm{n}$ & 21 March 2005 & 7 & 27 & 21 March 2005 & 16 & 26 & 23 March 2005 & 13 & 5 \\
\hline $\mathrm{n}$ & 07 May 2005 & 19 & 17 & 08 May 2005 & 17 & 44 & 10 May 2005 & 8 & 6 \\
\hline $\mathrm{y}$ & 15 May 2005 & 4 & 23 & 15 May 2005 & 8 & 58 & 17 May 2005 & 10 & 25 \\
\hline $\mathrm{n}$ & 20 May 2005 & 3 & 19 & 20 May 2005 & 7 & 18 & 21 May 2005 & 6 & 45 \\
\hline $\mathrm{y}$ & 29 May 2005 & 9 & 59 & 30 May 2005 & 2 & 3 & 30 May 2005 & 23 & 55 \\
\hline $\mathrm{n}$ & 12 June 2005 & 8 & 11 & 12 June 2005 & 17 & 7 & 13 June 2005 & 15 & 42 \\
\hline $\mathrm{y}$ & 14 June 2005 & 18 & 35 & 15 June 2005 & 6 & 7 & 16 June 2005 & 8 & 49 \\
\hline $\mathrm{y}$ & 10 July 2005 & 3 & 44 & 10 July 2005 & 11 & 52 & 12 July 2005 & 5 & 47 \\
\hline $\mathrm{y}$ & 16 July 2005 & 2 & 36 & 16 July 2005 & 5 & 39 & 16 July 2005 & 17 & 35 \\
\hline $\mathrm{y}$ & 17 July 2005 & 1 & 50 & 17 July 2005 & 8 & 44 & 18 July 2005 & 6 & 58 \\
\hline $\mathrm{n}$ & 23 August 2005 & 20 & 11 & 24 August 2005 & 1 & 13 & 24 August 2005 & 6 & 19 \\
\hline $\mathrm{y}$ & 02 September 2005 & 14 & 24 & 02 September 2005 & 19 & 12 & 03 September 2005 & 5 & 6 \\
\hline $\mathrm{y}$ & 09 September 2005 & 14 & 7 & 09 September 2005 & 21 & 59 & 10 September 2005 & 4 & 12 \\
\hline $\mathrm{y}$ & 11 September 2005 & 1 & 15 & 11 September 2005 & 5 & 32 & 12 September 2005 & 6 & 22 \\
\hline $\mathrm{y}$ & 12 September 2005 & 6 & 22 & 12 September 2005 & 20 & 34 & 13 September 2005 & 7 & 19 \\
\hline $\mathrm{n}$ & 13 September 2005 & 7 & 19 & 13 September 2005 & 9 & 52 & 14 September 2005 & 11 & 41 \\
\hline $\mathrm{y}$ & 15 September 2005 & 8 & 52 & 15 September 2005 & 15 & 30 & 16 September 2005 & 10 & 2 \\
\hline $\mathrm{n}$ & 31 October 2005 & 0 & 26 & 31 October 2005 & 2 & 49 & 31 October 2005 & 22 & 3 \\
\hline $\mathrm{n}$ & 31 December 2005 & 0 & 21 & 31 December 2005 & 6 & 21 & 01 January 2006 & 14 & 7 \\
\hline $\mathrm{y}$ & 01 January 2006 & 14 & 7 & 01 January 2006 & 15 & 4 & 02 January 2006 & 3 & 22 \\
\hline $\mathrm{n}$ & 05 February 2006 & 18 & 52 & 05 February 2006 & 19 & 50 & 06 February 2006 & 11 & 57 \\
\hline $\mathrm{y}$ & 13 April 2006 & 12 & 13 & 13 April 2006 & 15 & 30 & 14 April 2006 & 11 & 5 \\
\hline $\mathrm{y}$ & 09 July 2006 & 21 & 28 & 10 July 2006 & 21 & 16 & 11 July 2006 & 18 & 36 \\
\hline $\mathrm{y}$ & 19 August 2006 & 11 & 45 & 20 August 2006 & 14 & 7 & 21 August 2006 & 16 & 44 \\
\hline $\mathrm{n}$ & 30 August 2006 & 13 & 43 & 31 August 2006 & 7 & 7 & 01 September 2006 & 8 & 28 \\
\hline $\mathrm{n}$ & 30 September 2006 & 6 & 7 & 30 September 2006 & 9 & 13 & 30 September 2006 & 20 & 38 \\
\hline $\mathrm{n}$ & 17 November 2006 & 21 & 45 & 18 November 2006 & 9 & 7 & 20 November 2006 & 3 & 12 \\
\hline $\mathrm{n}$ & 29 November 2006 & 3 & 29 & 29 November 2006 & 5 & 46 & 30 November 2006 & 14 & 14 \\
\hline $\mathrm{y}$ & 08 December 2006 & 4 & 33 & 09 December 2006 & 6 & 7 & 09 December 2006 & 14 & 17 \\
\hline $\mathrm{y}$ & 14 December 2006 & 14 & 7 & 14 December 2006 & 22 & 44 & 15 December 2006 & 12 & 29 \\
\hline $\mathrm{y}$ & 16 December 2006 & 17 & 57 & 17 December 2006 & 0 & 58 & 17 December 2006 & 16 & 3 \\
\hline $\mathrm{n}$ & 14 January 2007 & 9 & 11 & 14 January 2007 & 12 & 48 & 15 January 2007 & 7 & 4 \\
\hline $\mathrm{y}$ & 19 November 2007 & 18 & 5 & 20 November 2007 & 0 & 13 & 20 November 2007 & 12 & 27 \\
\hline $\mathrm{n}$ & 17 September 2008 & 1 & 13 & 17 September 2008 & 4 & 54 & 18 September 2008 & 8 & 44 \\
\hline $\mathrm{n}$ & 04 December 2008 & 4 & 6 & 04 December 2008 & 14 & 19 & 05 December 2008 & 11 & 29 \\
\hline $\mathrm{n}$ & 16 December 2008 & 22 & 14 & 17 December 2008 & 4 & 40 & 17 December 2008 & 17 & 26 \\
\hline
\end{tabular}

